Check for updates

Cite this: RSC Adv., 2018, 8, 19939

Received 19th February 2018

Accepted 14th May 2018

DOI: $10.1039 / c 8 r a 01513 a$

rsc.li/rsc-advances

\section{Bottom-electrode induced defects in self- assembled monolayer (SAM)-based tunnel junctions affect only the SAM resistance, not the contact resistance or SAM capacitance $\dagger$}

\begin{abstract}
C. S. Suchand Sangeeth, $\stackrel{+}{+}^{a}$ Li Jiang $^{a}$ and Christian A. Nijhuis (DD *abc
In large area molecular junctions, defects are always present and can be caused by impurities and/or defects in the electrode materials and/or SAMs, but how they affect the electrical characteristics of junctions has rarely been studied. Usually, junctions are characterized by two-terminal current-voltage measurements where only the total current across the junction is measured, but with these methods one cannot distinguish how the individual components of the junctions are altered by the defects. Here we show that the roughness of the bottom-electrode is a crucial factor in determining the electrical properties of self-assembled monolayer (SAM)-based junctions. We used potentiodynamic impedance spectroscopy to reveal which components of the junctions are altered by defective bottom electrodes because this method allows for direct determination of all components that impede charge transport in the equivalent circuit of the junctions. We intentionally introduced defects via the roughness of the bottom electrode and found that these defects lower the SAM resistance but they do not alter the capacitance of the SAM or the contact resistance of the junction. In other words, defective junctions can be seen as "leaky capacitors" resulting in an underestimation of the SAM resistance of two orders of magnitude. These results help to improve the interpretation of data generated by SAM-based junctions and explain in part the observed large spread of reported tunneling rates for the same molecules measured across different platforms.
\end{abstract}

\section{Introduction}

Molecular electronic devices can be divided into two classes: single molecule junctions ${ }^{1-5}$ and junctions based on a layer of molecules with a thickness of exactly one molecule. ${ }^{6-8}$ Molecular junctions based on self-assembled monolayers (SAMs) are appealing because they operate in the tunneling regime and therefore have complementary properties with respect to conventional devices, ${ }^{6,9-11}$ and, unlike single molecule junctions ${ }^{2-4}$ they can be fabricated on large scales and have found commercial applications, ${ }^{12}$ and the SAM structure can be characterized in detail. ${ }^{9,13-15}$ These SAM-based junctions are

\footnotetext{
aDepartment of Chemistry, National University of Singapore, 3 Science Drive 3, Singapore 117543, Singapore. E-mail: chmnca@nus.edu.sg; Fax: +656779 1691; Tel: +6565162667

${ }^{b}$ Centre for Advanced $2 D$ Materials and Graphene Research Centre, National University of Singapore, 6 Science Drive 2, Singapore 117546, Singapore

'NUSNNI-Nanocore, National University of Singapore, Singapore 117411, Singapore $\dagger$ Electronic supplementary information (ESI) available: Additional experimental details, Nyquist plots and plots of equivalent circuit parameters as a function BV. See DOI: 10.1039/c8ra01513a

‡ Current address: Department of Physics, National Institute of Technology Calicut, Kerala 673601, India.
}

usually fabricated by first forming a SAM on a metal bottomelectrode followed by the fabrication of a top-electrode on the SAM in a second step. ${ }^{14,16}$ Although most studies focus on optimizing the second step of the fabrication process, ${ }^{17-20}$ we $^{21-23}$ and Whitesides et al. ${ }^{24}$ have shown that defects in the junctions induced by the defects in the bottom-electrode greatly affect the electrical properties of SAM-based junctions. For instance, by systematically investigating the electrical properties of SAM-based junctions as a function of the surface roughness of the bottom-electrode, we found that the quality of the tunneling barrier defined by the SAM ${ }^{22}$ or the performance of a molecular diode ${ }^{21}$ depends strongly on the topography of the bottom electrode (see below). Defect free junctions cannot be made and, to a certain degree, defects will be always present due to unavoidable imperfections caused by defects in the electrode materials, e.g., step-edges, grain boundaries, or impurities, or phase domains in the SAMs..$^{21,22,24-26}$ We note that usually in single molecule experiments only ideal structures are considered and that the role of "defects" or non-ideal structures in these systems are difficult to determine. ${ }^{27-31}$ Thus understanding how defects affect the electrical properties of junctions, or cause junctions to fail, is important in the rational 
a)

\section{ideal junction}

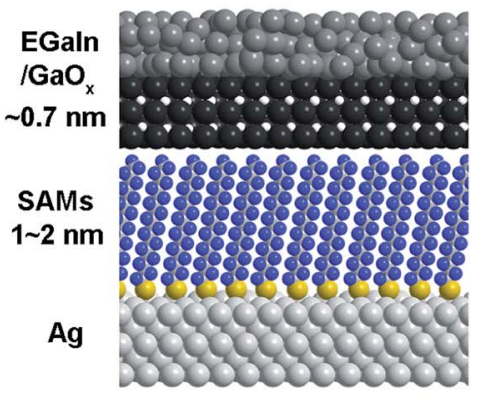

b)

$$
\text { defective junction }
$$

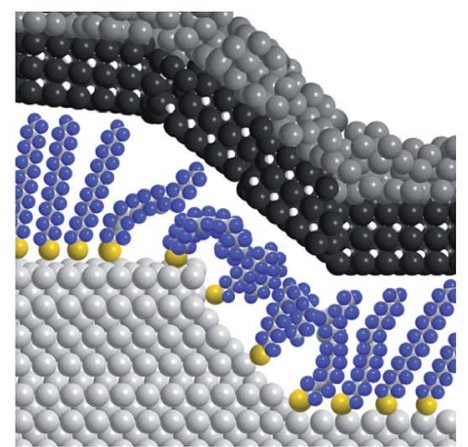

c) Equivalent circuit:

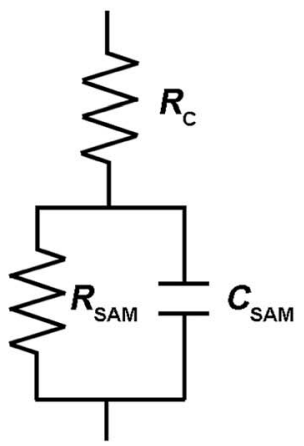

Fig. 1 Schematic illustration of an ideal junction without defects (a) and a junction with a disordered SAM on a defect in the bottom-electrode (b). (c) The equivalent circuit of the junction.

design of molecular junctions and interpretation of charge transport data they generate.

In previous works, we have only pointed out that defects are important and change the apparent values of $\beta,^{22}$ or introduce leakage currents reducing the function of molecular diodes, ${ }^{21}$ here we report which parts of the junctions are affected by defects revealed by impedance spectroscopy. The central question we wish to address here is: which part(s) of the junction, or more specifically, which circuit element(s), is (are) sensitive to defects inside the junction induced by the defects in the bottom-electrode and how will the presence of defects affect the interpretation of charge transport data obtained from SAMbased junctions? The key finding of this work is that imperfections in the bottom-electrode material only affect the SAM resistance whilst other observables associated with typical tunneling behavior (e.g., exponential decay of the current with molecular length) and good quality of the junctions (e.g., high yields), and other circuit components (the capacitance and contact resistance of the junctions) are not affected by defects. Our results show that defective junctions with bottomelectrodes obtained by commonly used direct deposition methods behave as "leaky capacitors" resulting in an underestimation of the SAM resistance by two orders of magnitude. Therefore, this work will be useful for future quantitative analyses of charge transport rates across platforms which has been notoriously difficult (see for a detailed review ref. 14).

Mostly, the electrical properties of SAM based-tunnel junctions are probed with DC conductance measurements which only determine the total current flowing across a junction as a function of applied bias. ${ }^{32-34}$ DC experiments alone cannot reveal the role of individual junction components (the two SAM-electrode interfaces and the SAM) and usually have to be combined with systematical studies involving changes of the electrode materials or chemical modifications to the SAMs.,34-41 We note that here we measured the contact resistance directly, without the need for long extrapolations to $n=0$, or fitting the low bias linear part of the $J(V)$ curve. In previous works we have compared our values of the contact resistance, and that obtained by others, ${ }^{33,42}$ and recently we directly compared the contact resistance of EGaIn junctions and conductive probe atomic force microscopy based junctions. ${ }^{43}$ Here the major difference is that EGaIn junctions have a 1-2 orders of magnitude higher contact resistance due to the presence of the $\mathrm{GaO}_{x}$ layer which has indeed a 1-2 orders of higher resistance than bulk EGaIn ${ }^{33,42-44}$ Fig. 1 shows a SAM-based junction schematically along with an equivalent circuit that describes such a junction consisting of the contact resistance of the SAM $\left(R_{\mathrm{C}}\right)$ with both electrodes in series with a parallel combination of the SAM resistance $\left(R_{\mathrm{SAM}}\right)$ and capacitance $\left.\left(C_{\mathrm{SAM}}\right)\right)^{33,42}$ This equivalent circuit has been used to model other SAM-based junctions as well. ${ }^{45}$ We have shown before that frequency dependent impedance measurements make it possible to determine $R_{\mathrm{C}}$, $R_{\mathrm{SAM}}$, and $C_{\mathrm{SAM}}$, and thus to separate the individual components that contribute to charge transport from one and the other..$^{33,46,47}$ As depicted in Fig. 1, the top-electrode in our studies was fabricated using the "EGaIn-technique" and consists of a eutectic alloy of Ga and In (EGaIn) coated with a self-limiting layer of predominantly $\mathrm{Ga}_{2} \mathrm{O}_{3}$ of $0.7 \mathrm{~nm}$ thick. ${ }^{33,48}$ Here, the top electrode forms an ohmic contact with the SAM and the resistance of the $\mathrm{GaO}_{x}$ layer and the $\mathrm{SAM} / /$ top electrode contact (// is a van der Waals interface) are the main contributor to $R_{\mathrm{C}} \cdot{ }^{33,42,48}$ The junction itself can be modelled as a parallel plate capacitor through which a tunneling current flows. ${ }^{23}$

The magnitude of the tunneling current density, $J$ (in A $\mathrm{cm}^{-2}$ ), flowing across junctions with SAMs of, for instance, $\mathrm{S}\left(\mathrm{CH}_{2}\right)_{n-1} \mathrm{CH}_{3}$ (or in short $\mathrm{SC}_{n}$ where $n$ is the number of $\mathrm{CH}_{2}$ units) is mostly analyzed with the general tunneling equation (eqn (1))

$$
J=J_{0}(V) \mathrm{e}^{-\beta d_{\mathrm{SAM}}}=J_{0}(V) 10^{-\beta d_{\mathrm{SAM}} / 2.303}
$$

where the value of $J$ at a given bias is plotted against the thickness (or the length of the molecules) of the SAM, $d_{\mathrm{SAM}}$ (in $\mathrm{n}$ ), and the graph is fitted to eqn (1). The slope of this fit gives the tunneling decay coefficient, $\beta\left(\mathrm{n}^{-1}\right)$, and the intercept with the $y$-axis gives the pre-exponential factor $J_{0}$ (in $\mathrm{A} \mathrm{cm}^{-2}$ ). Recently, we have shown that the value of $\beta$ decreases from 1.0 $\mathrm{n}^{-1}$ (which is associated with a high quality tunnel junctions) 
obtained from junctions with an ultra-flat bottom electrodes, to as low as $0.41 \mathrm{n}^{-1}$ for the same junction but with rough bottom electrodes. ${ }^{22}$ We also found that the value of $J_{0}$ increases by three orders of magnitude with increasing geometrical junction area, but remains constant when the junction area was smaller than $9.6 \times 10^{2} \mu \mathrm{m}^{2} .^{23}$ In EGaIn junctions, very thin SAMs $(n<$ 10), which have liquid-like characteristics and thus intrinsic disorder, ${ }^{49}$ still have values of $\beta$ close $1 \mathrm{n}^{-1}$ indicating that the changes in the electrical behavior of the junctions is not caused by changes in molecule-electrode couplings, but by defects which change the effective tunneling distance. ${ }^{50}$ From these studies we conclude that the presence of thin area defects, which reduce the effective value of $d_{\mathrm{SAM}}$ and therefore cause high local currents at these defect sites, add complexity to the interpretation of the data which are not straightforwardly captured by fitting the charge transport data to eqn (1)..$^{22,23}$

How defects alter the electrical properties of tunnel junctions have been often characterized in tunnel junctions based on metal oxides, ${ }^{51-54}$ but not in SAM-based junctions. Based on eqn (1), defective sites in junctions can be classified into two categories: defects that increase (thick area defects) or decrease (thin area defects) the effective electrode-electrode distance. ${ }^{24,55}$ In EGaIn junctions, large parts within the junction area are thick area defects due to the surface roughness of the EGaIn top electrode $;^{50}$ these areas do not contribute significantly to the measured value of $J$ as the current flowing across such defect sites are very low. In contrast, the current flowing across thin area defects can be very high and even dominate the measured value of $J^{56-59}$ and can be caused by, e.g., grain boundaries, chemi- or physisorbed impurities, or phase domains of the SAMs. ${ }^{21,23-26}$ Here, we used frequency dependent (ranging from $1 \mathrm{~Hz}$ to $1 \mathrm{MHz}$ ) impedance measurements on tunneling junctions with alkanethiolate SAMs ( $\mathrm{SC}_{n}$ with $n=10,14$ or 18) formed on $\mathrm{Ag}$ substrates with different topographies. By varying the surface topography we effectively varied the root mean square (rms) surface roughness, the grain size, and area of grain boundaries $\left(A_{\mathrm{gb}}\right)$. To capture all these variables in a single parameter, we used the bearing volume (BV) given by eqn (2) where $N_{\mathrm{gr}}$ is the number of grains. ${ }^{22,60}$

$$
\mathrm{BV}=N_{\mathrm{gr}} \times A_{\mathrm{gb}} \times \mathrm{rms}
$$

In terms of $\mathrm{BV}$ values, the more defective the bottomelectrodes are, the higher the BV values are. Since we used a top-electrode with an area of $9.6 \times 10^{2} \mu \mathrm{m}^{2}$ for the electrical measurements of all the junctions, we effectively varied the number of defects inside the tunneling junction by changing BV. We found that defects inside the junctions only affect the SAM resistance, but not the capacitance or the contact resistance of the junction.

\section{Experimental section}

\section{Preparation of $\mathrm{Ag}$ surfaces}

We followed previously reported procedures to prepare the four types of Ag surfaces with different surface topographies. ${ }^{22,60}$ Generally, we used high grade silver with purity of $99.999 \%$
(Super Conductor Material, Inc (USA)) and one-side polished silicon wafers (University Wafers, USA) as the template for templated-stripped (TS) Ag surfaces, or as substrates for $\mathrm{Ag}$ surfaces obtained by direct evaporation (DE). The $\mathrm{Ag}$ was deposited at a rate of $0.5 \AA \mathrm{s}^{-1}$ for the first $50 \mathrm{~nm}$ and followed by the deposition of $250 \mathrm{~nm}$ at a rate of $1 \AA^{-1}$ (at a base pressure of $2-5 \times 10^{-6}$ bar) in the case of $\mathrm{Ag}^{\mathrm{A}-\mathrm{TS}}, \mathrm{Ag}^{\mathrm{TS}}$, and $\mathrm{Ag}^{\mathrm{DE}, 1}$, surfaces. The $\mathrm{Ag}^{\mathrm{DE}, 2}$ surfaces were obtained by deposition of $\mathrm{Ag}$ a rate of $4 \AA \mathrm{s}^{-1}$. After deposition of $\mathrm{Ag}$, the $\mathrm{Ag}^{\mathrm{DE}, 1}$ and $\mathrm{Ag}^{\mathrm{DE}, 2}$ substrates were used as such. To obtain the $\mathrm{Ag}^{\mathrm{A}-\mathrm{TS}}$ surfaces, after metal deposition the substrates were annealed at $200{ }^{\circ} \mathrm{C}$ (the temperature was increased from room temperature at a rate of $15{ }^{\circ} \mathrm{C} \mathrm{min}{ }^{-1}$ ) for $30 \mathrm{~min}$ under vacuum inside the vacuum chamber of the thermal evaporate. After annealing, we cooled down the substrates to room temperature overnight inside the UHV chamber. We applied an optical adhesive (OA, Norland, no. 61) on the Ag substrates to glue the glass supports $\left(2 \times 3 \mathrm{~cm}^{2}\right.$, cleaned by immersion into piranha solution $\left(\mathrm{H}_{2}-\right.$ $\mathrm{SO}_{4}: \mathrm{H}_{2} \mathrm{O}_{2}=3: 1$ ) for $20 \mathrm{~min}$, followed by washing with $\mathrm{H}_{2} \mathrm{O}$ and dried in a stream of $\mathrm{N}_{2}$ gas. The size of the glass supports will be the final dimension of the bottom-electrode. Note, we used Ag surfaces in this study since we can generate surfaces with large changes in the $\mathrm{BV}$ values as reported previously. ${ }^{21,22,60,61}$ These glass supports were further cleaned with air plasma for $2 \mathrm{~min}$ and then glued to the metal $\mathrm{Ag}$ surfaces. We cured the $\mathrm{OA} / \mathrm{Ag} /$ silicon substrates under UV light (100 watt using a ultraviolet light at a distance of $60 \mathrm{~cm}$ from the substrates to minimize heating) for 1 hour. After curing, the glass/AO/Ag stacks were stripped of the template just before use. The $\mathrm{Ag}^{\mathrm{TS}}$ surfaces were obtained in the same way as described above to obtain the $\mathrm{Ag}^{\mathrm{A}-\mathrm{TS}}$ but without the annealing step.

\section{Atomic force microscopy}

Atomic force microscopy (AFM) images were recorded on a Bruker Dimension FastScan AFM by using tapping mode tips with intermittent contacts (model: FASTSCAN-A, resonant frequency: $14 \mathrm{MHz}$, and force constant: $18 \mathrm{~N} \mathrm{~m}^{-1}$ ).

\section{Preparation of the SAMs}

We purified the $n$-alkanethiols (Sigma Aldrich) by recrystallization from ethanol (AR grade; three times) before use because their purity, as received, ranged from 95 to $98 \% .^{22,61}$ Since thiols can decompose and form disulfides in ambient conditions, we further purified the as received $n$-alkanethiols (Sigma-Aldrich) prior to use. The $n$-alkanethiols ( $n=10,14$ or 18 ) were recrystallized from ethanol (AR grade) under an atmosphere of $\mathrm{N}_{2}$ at $-20{ }^{\circ} \mathrm{C}$ followed by a quick filtration. We repeated the recrystallization of each compound for 3 times, and we stored the purified thiols under $\mathrm{N}_{2}$ at $4{ }^{\circ} \mathrm{C}$. We followed a previously reported protocol to prepare the SAMs. ${ }^{22}$ First, we prepared $3 \mathrm{mM}$ ethanolic solutions of each alkanethiol and purged the solutions with $\mathrm{N}_{2}$ gas for at least 15 min prior to immersion of the $\mathrm{Ag}$ substrates. The SAMs were formed over a period of three hours in the ethanolic solutions under inert atmosphere to prevent oxidation of the $\mathrm{M}-\mathrm{S}$ bond and avoid the formation of silver oxides. The $\mathrm{Ag}^{\mathrm{DE}, 1}$ and $\mathrm{Ag}^{\mathrm{DE}, 2}$ substrates were immersed into the 
solution immediately after they were removed from the vacuum chamber of the thermal evaporator and the $\mathrm{Ag}^{\mathrm{A}-\mathrm{TS}}$ and $\mathrm{Ag}^{\mathrm{TS}}$ substrates were immersed into the solution immediately (within a few seconds) after template-stripping.

\section{Fabrication of the junctions}

We formed top-contacts on the SAM using $\mathrm{GaO}_{x} / \mathrm{EGaIn}$ confined in a through-hole in poly(dimethylsiloxane) (PDMS) using a method which has recently been reported by us. ${ }^{61}$ Here, the liquid-metal is injected in a micro-channel in PDMS; this microchannel was aligned over a through-hole which was connected to a second channel to which vacuum was applied so that the liquid-metal was forced into the though-hole. The slab of PDMS with the $\mathrm{GaO}_{x} / \mathrm{EGaIn}$ stabilized in the through-hole was gently placed on top of the SAM. After electrical characterization, we removed the top-electrode from the SAM to form a new junction. The same top-electrode could be used to form 20-25 junctions.

\section{Electrical characterization of the junctions}

The $J(V)$ measurements were carried out using a Keithley 6430 source meter and data were acquired using LabView 2010 following previously reported methods. ${ }^{61}$ Before we started the impedance measurements, we determined the $J(V)$ characteristics of the devices and recorded the values of $J$ over the range of biases of -0.50 to $0.50 \mathrm{~V}$ at intervals of $20 \mathrm{mV}$. We selected those junctions that had their electrical characteristic within one log-standard deviation from the Gaussian log-mean values of $J$ (which are reported in ref. 22) for the impedance measurements. All impedance measurements were repeated 3 times using 3 different junctions following previously reported procedures reported elsewhere. We measured the impedance of these junctions using a Solartron impedance analyzer (model Solartron 1260A with 1296A dielectric interface) in reference mode with a standard $10 \mathrm{pF}$ capacitor as the external reference (see ref. 33 and 42 for all the details). The potentiodynamic impedance measurements were conducted by applying an AC voltage of $30 \mathrm{mV}$ superimposed on a DC bias voltage and the frequency was varied from $1 \mathrm{~Hz}$ to $1 \mathrm{MHz}$ with 10 frequencies per decade. The geometrical junction areas was $9.6 \times 10^{2} \mu \mathrm{m}^{2}$ in all impedance measurements.

\section{Results and discussion}

The bottom electrodes

We fabricated four different types of bottom-electrodes with varying surface topographies to investigate how defects induced by the bottom-electrode alters the electrical characteristics, more specifically, the complex impedance of the junctions following previously reported methods (see experimental section for details). ${ }^{22,60}$ Fig. 2 shows the atomic force microscopy (AFM) images of the four Ag surfaces. These surfaces have different values of the root mean square (rms) surface roughness, number of grains $\left(N_{\mathrm{gr}}\right)$, and area of exposed grain boundaries $\left(A_{\mathrm{gb}}\right)$, Table $\mathrm{S} 1 \uparrow$ summarizes these parameters, and we used the bearing volume (BV in $\mathrm{nm}^{3}$; eqn (2)) to compare the topography of these bottom-electrodes. Low BV values indicate the surface is smooth and has low density of defects, while large BV values indicate the surface is dominated by the defects. In agreement with our previous a)

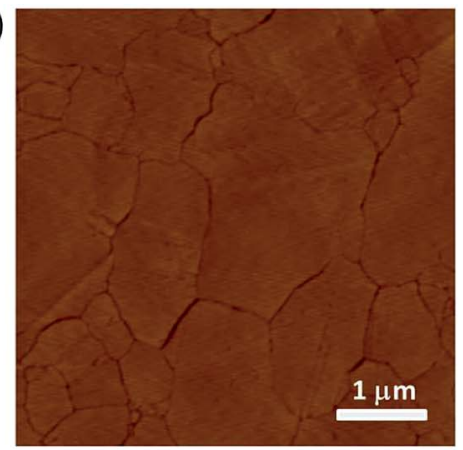

c)

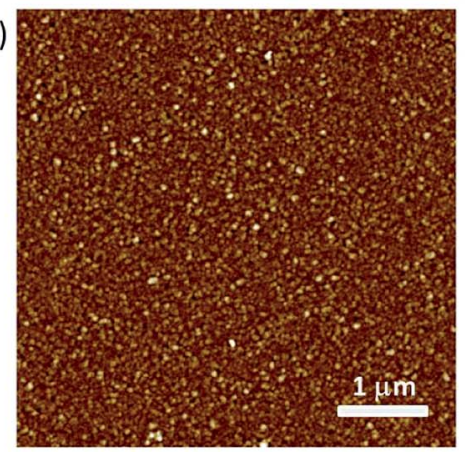

b)

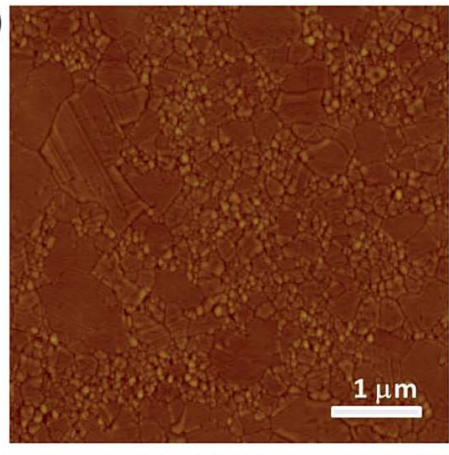

d)

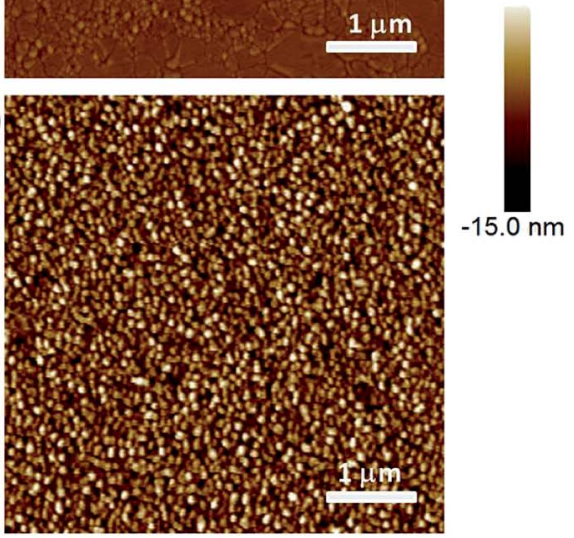

$15.0 \mathrm{~nm}$

Fig. 2 AFM images of the $\operatorname{Ag}^{\mathrm{A}-\mathrm{TS}}$ (a), $\mathrm{Ag}^{\mathrm{TS}}$ (b), $\mathrm{Ag}^{\mathrm{DE}, 1}$ (c), and $\mathrm{Ag}^{\mathrm{DE}, 2}$ (d) bottom electrodes. 

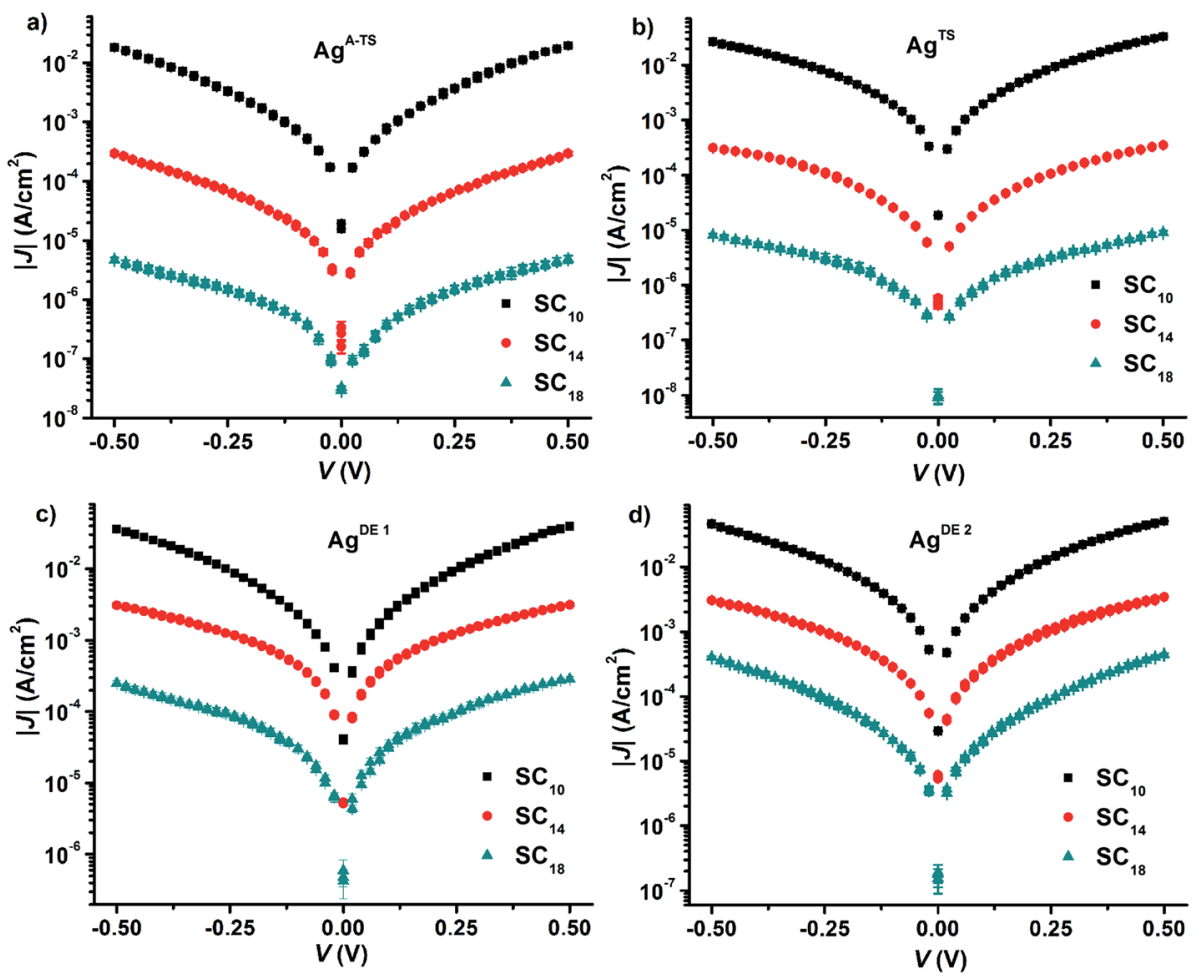

Fig. 3 The arithmetic average $J(V)$ characteristics of the junction with $\mathrm{SAMS}_{\mathrm{S}} \mathrm{SC}_{10}, \mathrm{SC}_{14}$, and $\mathrm{SC}_{18}$, supported by $\mathrm{Ag}^{\mathrm{A}-\mathrm{TS}}$ (a), $\mathrm{Ag}^{\mathrm{TS}}$ (b), $\mathrm{Ag}^{\mathrm{DE}, 1}$ (c), or $\mathrm{Ag}^{\mathrm{DE}, 2}(\mathrm{~d})$, bottom electrodes. The error bars represent the standard deviation obtained from three different junctions.

work, $\mathrm{Ag}^{\mathrm{A}-\mathrm{TS}}$ and $\mathrm{Ag}^{\mathrm{TS}}$ surfaces have low BV values of $8 \times 10^{4}$ $\mathrm{nm}^{3}$ and $1 \times 10^{5}$, respectively, while that of the $\mathrm{Ag}^{\mathrm{DE}, 1}$ and $\mathrm{Ag}^{\mathrm{DE}, 2}$ surfaces are more than two orders of magnitude larger $\left(\sim 2 \times 10^{6} \mathrm{~nm}^{3}\right.$ and $\sim 3 \times 10^{6} \mathrm{~nm}^{3}$, respectively).

This large difference in the $\mathrm{BV}$ values is also visible by eye in Fig. 2. The $\mathrm{Ag}^{\mathrm{A}-\mathrm{TS}}$ surface only consists of large grains separated by wide grain boundaries, while the $\mathrm{Ag}^{\mathrm{TS}}$ surface consists of large grains separated by small grains. Here the effect of annealing is clearly visible as the small grains are effectively removed. In addition, since both types of surfaces were obtained from template-stripping, all the grains are in the same plane defined by the template. In sharp contrast, both types of $\mathrm{Ag}^{\mathrm{DE}}$ surfaces have a large surface roughness because the grains are small and are out of plane with respect to each other. Therefore, the $\mathrm{Ag}^{\mathrm{DE}}$ surfaces have a large surface fraction of exposed grain boundaries at which SAMs cannot pack well while both types of $\mathrm{Ag}^{\mathrm{TS}}$ surfaces have a relatively small amount of exposed grain boundaries.

\section{DC characterization of the junctions}

We formed the junctions using a previously reported procedure. ${ }^{61}$ Briefly, we formed SAMs of $n$-alkanethiolate SAMs $\left(\mathrm{S}_{(}\left(\mathrm{CH}_{2}\right)_{n-1} \mathrm{CH}_{3} \equiv \mathrm{SC}_{n}\right.$ with $n=10,14$, or 18$)$ supported by the four types of silver surfaces. Next, we formed electrical contacts to these SAMs with EGaIn. We used $\mathrm{GaO}_{x} / \mathrm{EGaIn}$ top-electrodes stabilized in a through-hole in PDMS because these junctions are mechanically stable and suitable to perform the potentiodynamic impedance spectroscopy measurements which took about $2 \mathrm{~h}$ per junction to complete.
Before we started the impedance measurements, we recorded $J(V)$ curves to confirm the junctions had their electrical characteristics within one log-standard deviation from the Gaussian log-mean $J(V)$ curves reported previously. ${ }^{22}$ Fig. 3 shows the $J(V)$ curves recorded from the junctions. Here, we repeated our experiments three times and the $J(V)$ curves in Fig. 3 are the arithmetic mean and the error bar represents the standard deviations. From these data, we derived for each type of junction the value of $\beta$ and $J_{0}$ by plotting the values of $\langle|J|\rangle$ determined at $-0.50 \mathrm{~V}$ against the number of carbons in the backbone, $n$, of the SAM followed by fitting the general tunneling equation (eqn (1)) to these graphs. Fig. S1† shows the results and that indeed the current exponentially decays with increasing $n$. The values of $\beta$ are $1.03 \pm 0.04$ and $1.05 \pm$ $0.05 \mathrm{n}^{-1}$ for junctions with $\mathrm{Ag}^{\mathrm{A}-\mathrm{TS}}$ and $\mathrm{Ag}^{\mathrm{TS}}$ bottom electrodes which are close to the consensus value of $1.0 \mathrm{n}^{-1}$ associated with high quality junctions. In contrast, junctions with $\mathrm{Ag}^{\mathrm{DE}, 1}$ or $\mathrm{Ag}^{\mathrm{DE}, 2}$ bottom electrodes have low $\beta$ values of $0.54 \pm 0.05$ and $0.58 \pm 0.06 \mathrm{n}^{-1}$ which is associated with defective junctions and in agreement with our previous findings. ${ }^{22}$ Despite the different values of $\beta$, all junctions with $\mathrm{SC}_{10}$ SAMs have similar values of $J$. The difference in values of $J$ at $0.5 \mathrm{~V}$ as a function of $\mathrm{BV}$, however, is large (two orders of magnitude) for junctions with SAMs of $n=18$ as function of the type of surface. These observations have been recently explained by us and we found that junctions with thin liquid-like SAMs (here with $n=10$ ) are capable of self-repair and compensate for defects in the bottom-electrode material, but thick crystallinelike SAMs are not capable of this self-repair and therefore 

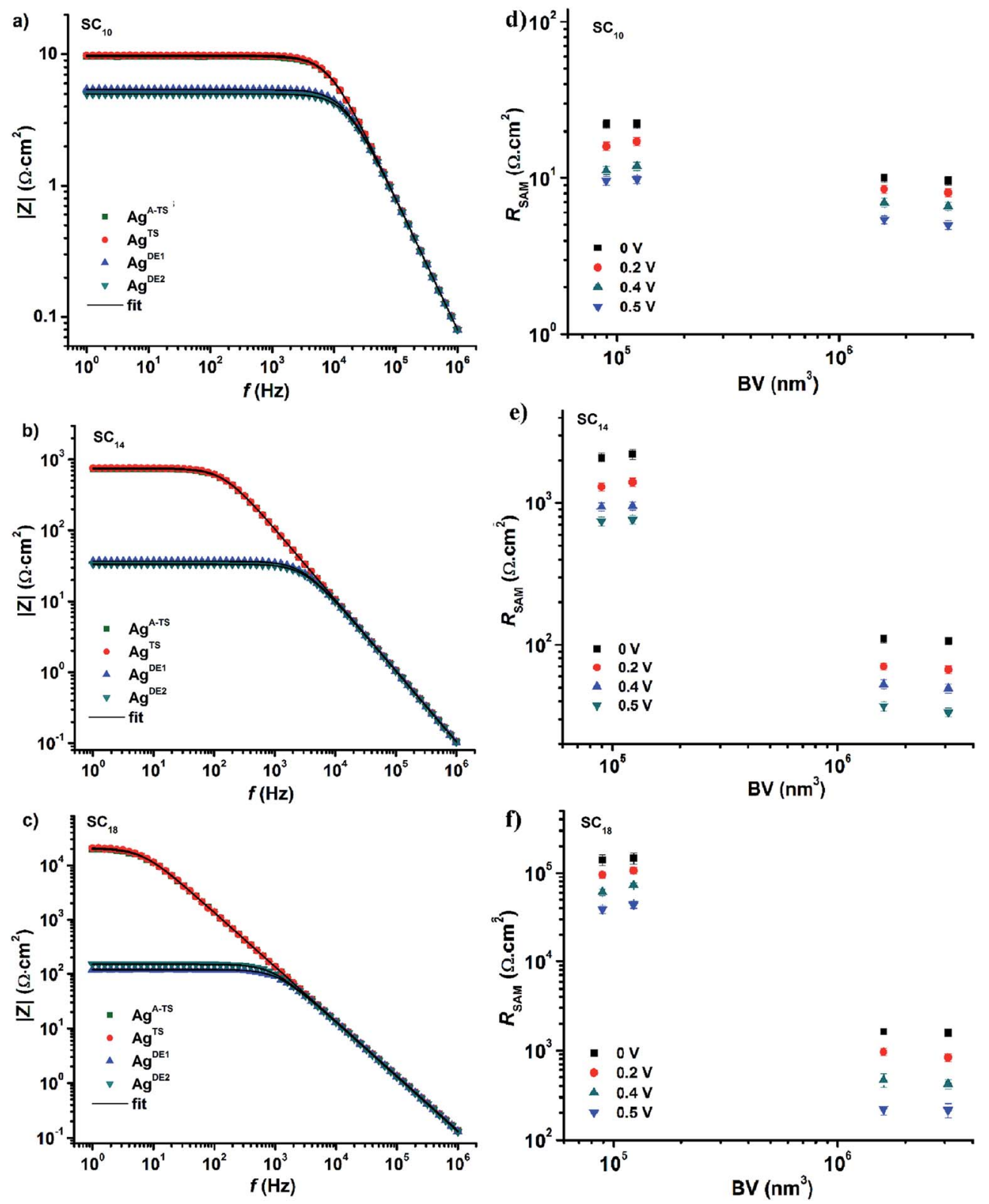

Fig. $4|Z|$ vs. frequency at $0.50 \mathrm{~V}$ obtained from junctions with $\mathrm{SAMs}$ of (a) $\mathrm{SC}_{10}$, (b) $\mathrm{SC}_{14}$, and (c) $\mathrm{SC}_{18}$, with different bottom electrodes as indicated. The black solid line is a fit of eqn (3) to the data. The values of RSAM for junction with $\mathrm{SC}_{1} \mathrm{O}$ (d), $\mathrm{SC}_{14}$ (e), and $\mathrm{SC}_{18}$ (f) vs. VB. Error bars represent the standard deviation obtained from three different junctions.

result in defective junctions with large leakage currents flowing at defect sites. ${ }^{25}$

\section{The equivalent circuit}

We have shown before that our junctions can be modelled with a simple equivalent circuit (Fig. 1c) consisting of $R_{\mathrm{SAM}}$ in parallel with $C_{\mathrm{SAM}}$ both in series with $R_{\mathrm{C}}{ }^{33,42}$ The complex impedance of this equivalent circuit is given by

$$
Z=\left(R_{\mathrm{C}}+\frac{R_{\mathrm{SAM}}}{1+\omega^{2} R_{\mathrm{SAM}}{ }^{2} C_{\mathrm{SAM}}{ }^{2}}\right)-j\left(\frac{\omega C_{\mathrm{SAM}} R_{\mathrm{SAM}}{ }^{2}}{1+\omega^{2} R_{\mathrm{SAM}}{ }^{2} C_{\mathrm{SAM}}{ }^{2}}\right)
$$

This equivalent circuit implies that SAMs behave as a dielectric placed between two parallel plates (the electrodes). The SAM impedes the current flowing through the junction with a complex impedance which is a parallel combination of $R_{\mathrm{SAM}}$ and $C_{\mathrm{SAM}}$. The SAM is coupled to the electrodes through a certain contact resistance $R_{\mathrm{C}}$ which is in series with the parallel RC circuit element.

The physical meaning of the equivalent circuit has been discussed before and is summarized here briefly. ${ }^{33,42}$ In EGaIn based molecular junctions the value of the contact resistance $R_{\mathrm{C}}$ is dominated by the $\mathrm{SAM} / / \mathrm{GaO}_{x}$ resistance. In our earlier work we reported that the contact resistances of the contact probes with the electrodes, the Ag-thiolate interface and the low 

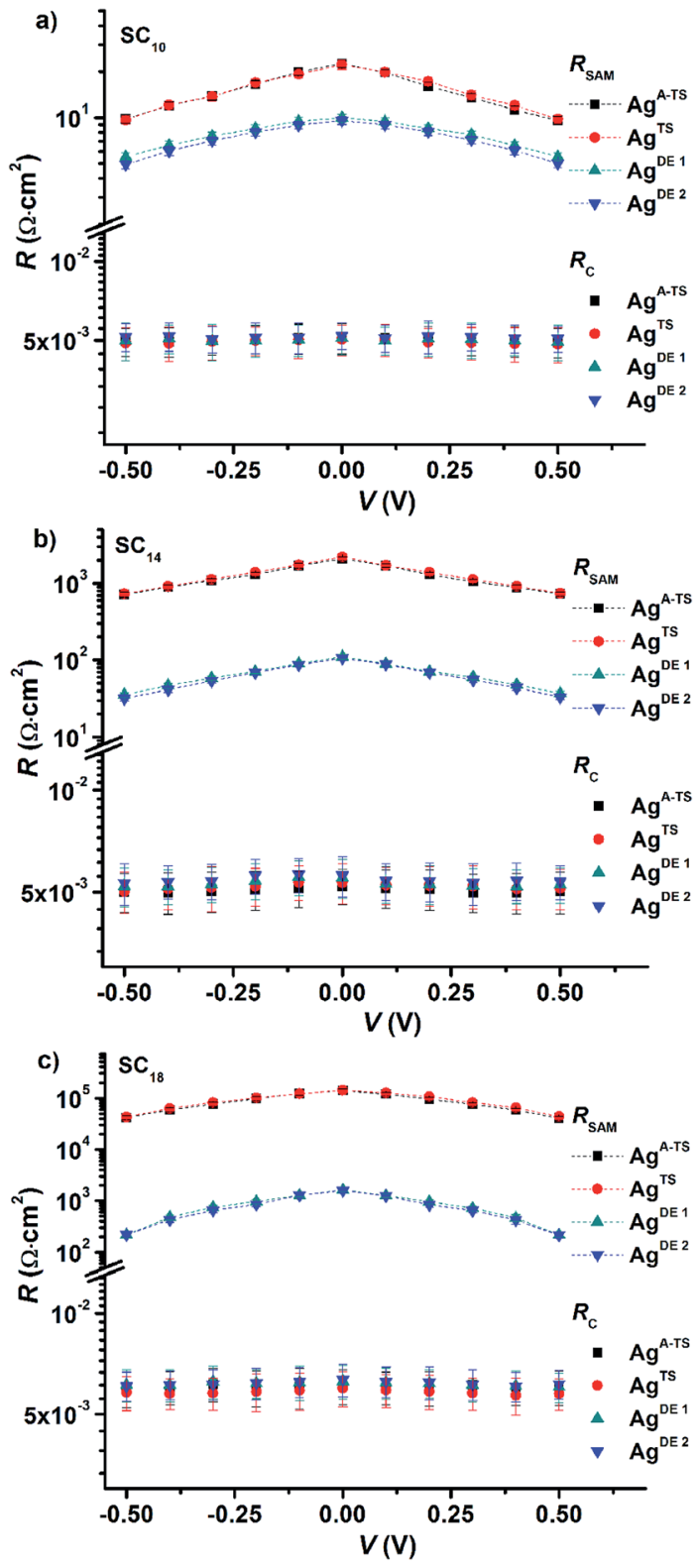

Fig. 5 The SAM resistance $\left(R_{\text {SAM }}\right)$ and contact resistance $\left(R_{C}\right)$ vs. DC bias voltages for junction with $\mathrm{SC}_{10} \mathrm{SAMs}(\mathrm{a}), \mathrm{SC}_{14} \mathrm{SAMs}(\mathrm{b})$, and $\mathrm{SC}_{18}$ SAMs (c). The error bars represent the standard deviation of three independent impedance measurements.

resistance of the $\mathrm{GaO}_{x}$ layer only adds about $2 \%$ to the measured value $R_{\mathrm{C}}{ }^{42}$ The resistance of the SAM is given by eqn (4) which relate $d_{\mathrm{SAM}}$ to $R_{\mathrm{SAM}}$, where $R_{\mathrm{SAM}, 0}$ is the hypothetical resistance across the junction for $d_{\mathrm{SAM}}=0 \mathrm{~nm}$.

$$
R_{\mathrm{SAM}}=R_{\mathrm{SAM}, 0} \mathrm{e}^{\beta d_{\mathrm{SAM}}}
$$

We interpret the impedance results and equivalent circuit modeling using Landauer tunneling model modified by including the contact resistance term which is associated with the coupling of the molecules to electrodes (eqn (5) $)^{62}$

$$
R_{\text {junction }}=\frac{h}{2 e^{2} M}+\frac{h}{2 e^{2} M} \frac{1-T}{T}=R_{\mathrm{C}}+R_{\mathrm{SAM}}
$$

where $e$ is the electron charge, $h$ is the Planck's constant, $T$ is the transmission probability, and $M$ is the number of conduction channels. ${ }^{62}$ For an ideal point contact $(T=1)$, the contact resistance is the inverse of the universal quantum conductance $G_{0}=2 e^{2} / \mathrm{h}$. Eqn (5) explains the series combination of $R_{\mathrm{C}}$ and $R_{\mathrm{SAM}}$ in the equivalent circuit. The value of $C_{\mathrm{SAM}}$ can be described by the expression (eqn (6)) for capacitance in parallel plate geometry, where $d_{\mathrm{SAM}}$ is the distance between the metal surfaces, $\varepsilon_{0}$ is the permittivity of the free space, $\varepsilon_{\mathrm{r}}$ is the dielectric constant of the SAM, and $A_{\text {geo }}$ is the geometrical area of the capacitor surfaces. ${ }^{42}$

$$
C_{\mathrm{SAM}}=\varepsilon_{0} \varepsilon_{\mathrm{r}} A_{\mathrm{geo}} / d_{\mathrm{SAM}}
$$

\section{Potentiodynamic impedance spectroscopy}

Impedance $(Z)$ is a more general concept resistance than since it contains both amplitude and phase information of the signal. ${ }^{33}$ As mentioned in the Introduction, impedance spectroscopy makes it possible to determine all circuit elements of our junctions. Here we determined the impedance spectra as a function of bias, i.e., potentiodynamic impedance spectroscopy, how the defects inside the junctions affect all circuit elements. The impedance measurements were carried out by superimposing a sinusoidal signal (in the frequency range of $1 \mathrm{~Hz}$ to $1 \mathrm{MHz}$ ) on a DC bias ranging from $-0.5 \mathrm{~V}$ to $0.5 \mathrm{~V}$ at intervals of $0.1 \mathrm{~V}$. To improve the signal-to-noise ratios, we took the average of five impedance measurements at each given bias. This procedure was repeated three times with different junctions and the error bars represent the standard deviation of these three measurements.

Fig. 4 shows the frequency dependency of the modulus of the complex impedance $(|Z|)$ for all junctions at $-0.5 \mathrm{~V}$ (so-called Bode plots). The value $|Z|$ is nearly constant at low frequencies (dominated by the resistance of the SAM), but decreases with increasing frequency as the capacitive reactance $X_{\mathrm{c}}$ decrease with frequency $\omega$ (capacitive reactance $X_{\mathrm{c}}=1 / \omega C_{\mathrm{SAM}}$ ). The Nyquist plots (Fig. S3 $\dagger$ ), the imaginary part of $Z\left(Z^{\prime \prime}\right)$ plotted against the real part of $Z\left(Z^{\prime}\right)$, show only one semicircle which corresponds to the presence of one capacitor element in the equivalent circuit (the phase is $90^{\circ}$ for a capacitor and appears as a semi-circle in the Nyquist plot). At high frequencies, the capacitive reactance becomes negligible and the impedance will saturate to the total contact resistance $R_{\mathrm{C}}$ which has a phase of $0^{\circ}$ and is independent of the frequency (ohmic behavior). Fig. 4 shows that $|Z|$ of the junctions with SAMs of $\mathrm{SC}_{18}$ decreases over two orders of magnitude when bottom-electrodes with large BV values are used (the $\mathrm{Ag}^{\mathrm{DE}, 1}$ and electrodes $\mathrm{Ag}^{\mathrm{DE}, 2}$ ) while $|Z|$ does not change significantly as function of BV for junctions with $\mathrm{SC}_{10}$ SAM. These observations agree with the $J(V)$ behavior described above and follow our recent findings that liquid-like SAMs smoothen out the thin area defects easily compared to the long chain crystalline like SAMs. ${ }^{25}$ 

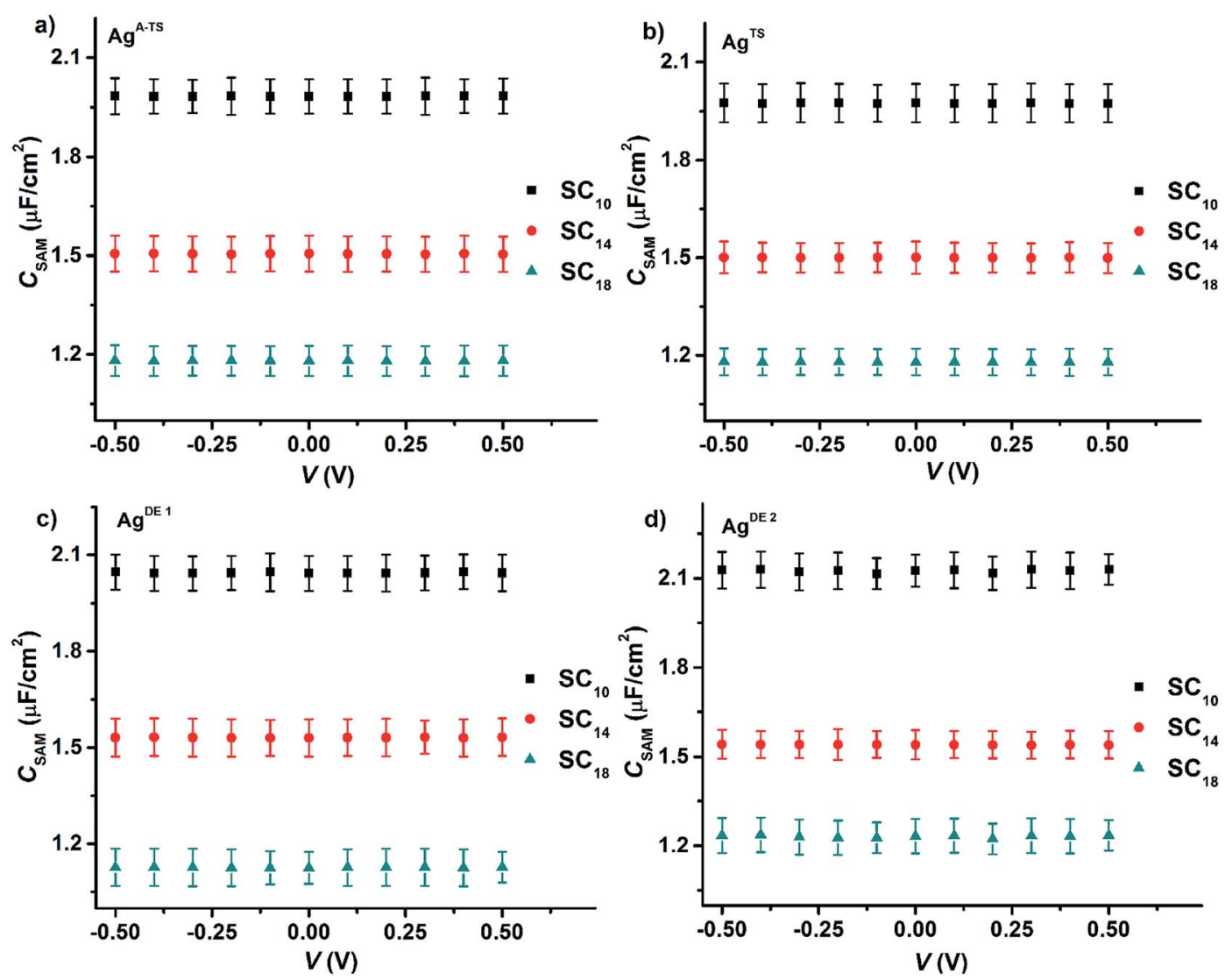

Fig. $6 C_{S A M}$ Vs. DC bias voltage for the junctions with SAMs on by $A^{A-T S}$ (a), $A^{T S}$ (b), $A^{D E, 1}$ (c), or $A^{D E, 2}$ (d), bottom electrodes. Error bars represent the standard deviation obtained from three different junctions.

Before we fitted our data to the equivalent circuit, we determined the quality of the impedance data using the KramersKronig transformation, or simply the so-called KK-test. ${ }^{63}$ The low $\chi_{\mathrm{KK}}^{2}$ values $\left(0.9 \times 10^{-3}\right.$ to $\left.1.2 \times 10^{-3}\right)$ indicate that the data are of good quality, no higher harmonics are present, and the junctions were stable during the measurements. Next, we fitted our data to eq. to extract the values of $R_{\mathrm{C}}, R_{\mathrm{SAM}}$, and $C_{\mathrm{SAM}}$. The solid black lines in Fig. 4 are fits to eqn (3) (with $\chi_{\mathrm{fit}}{ }^{2}$ in the range of $1.1 \times 10^{-3}$ to $1.5 \times 10^{-3}$ ) from which we conclude the equivalent circuit describes our junctions well. In the next section, we describe the circuit elements in more detail.

Fig. 5 shows the variation of $R_{\mathrm{SAM}}$ and $R_{\mathrm{C}}$ with DC bias voltage as a function of BV for different SAMs. The contact resistance $R_{\mathrm{C}}$ is independent of the applied DC bias and BV. These observations indicate that in all cases the contact resistance is ohmic in nature ${ }^{\mathbf{6 1}}$ and dominated by the van der Waals $\mathrm{SAM} / /$ top electrode contact which includes the resistance across the $\mathrm{GaO}_{x}$ layer. Our previous temperature dependent impedance studies on junctions with alkanethiolate SAMs on smooth electrodes showed that $R_{\mathrm{SAM}}, R_{\mathrm{C}}$, and $C_{\mathrm{SAM}}$, do not vary with temperature which indicates that the mechanism of charge transport in this system is to coherent tunneling. ${ }^{42}$ In the present study, in all cases the value of $R_{\mathrm{SAM}}$ decreases exponentially with increasing bias, in agreement with previously reported results. ${ }^{14,33}$ Therefore, regardless of the number of defects, both defective and good quality junctions show typical tunneling characteristics.

The value of $R_{\mathrm{SAM}}$ does not vary significantly as a function of $\mathrm{BV}$ for junctions with $\mathrm{SC}_{10}$ SAMs while it varies over two orders of magnitude for $\mathrm{SC}_{18}$ junctions. This observation can be explained as follows. We have suggested before that the thin liquid-like character of $\mathrm{SC}_{10}$ SAMs smoothen out the defects induced by the surface topography of the bottom electrode because the intramolecular van der Waals interactions are weak and so the molecules can relatively easily "fall over" and "fill in" defects. In contrast, thick crystalline-like $\mathrm{SC}_{18}$ SAMs cannot selfrepair as efficiently due to the high packing energy of the SAM (due to strong van der Waals interactions between the long alkyl chains). ${ }^{25}$ Therefore, the latter suffer from thin area defects that reduce the effective thickness of the SAM $\left(d_{\text {SAM }}\right)$ causing a large leakage current through the junction (see eqn (1)) while the former still forms good tunneling barriers even. Thus, the decrease in $R_{\mathrm{SAM}}$ with $\mathrm{BV}$ shows that the tunneling junctions formed on rough surfaces are dominated by thin area defects and that thin SAMs can compensate for defects over a wide range of $\mathrm{BV}$ values. The contact resistance is not noticeably changed because the area of defects is very small and charge conduction can be dominated even by a single pinhole. ${ }^{64}$ The presence of the defects in thick junctions is also apparent from the shape of the $R_{\mathrm{SAM}} v s$. $V$ curve which is "flatter" for junctions 
with $\mathrm{SC}_{18}$ than $\mathrm{SC}_{10}$ SAMs. This change in the shape can be related to conduction through pinholes where the current is concentrated and cause local Joule heating. ${ }^{23}$

Fig. 6 shows the values of $C_{\mathrm{SAM}}$ as a function voltage for all four junctions with different bottom electrode topographies and SAM thicknesses. The value of $C_{\mathrm{SAM}}$ shows no significant variation over the investigated $\mathrm{DC}$ bias range or value of $\mathrm{BV}$. By fitting a plot of $C_{\text {SAM }}$ against $1 / d_{\text {SAM }}$ to eqn (6) (Fig. S $2 \dagger$ ), we found that the value of $\varepsilon_{\mathrm{r}}$ of $3.2 \pm 0.3$ was the same within error for all junctions, in agreement with previously reported values. $^{33,45,65}$ Thus, the defects induced by the topography of the bottom electrode did not alter the dielectric constant of the SAMs inside the junctions.

\section{Conclusions}

Impedance spectroscopy is useful method to characterize how defects affect the junction characteristics

By far most studies of molecular junctions are based on twoterminal electrical measurements using DC methods. ${ }^{\mathbf{1 6 , 6 6 , 6 7}}$ Previously, we have shown that the yield in non-shorting junctions and reproducibility of the measurements (i.e., the log-standard deviations of the values of $J$ ) are remarkably insensitive to the topography of the bottom-electrode and hence these are poor indicators of the quality of these junctions. ${ }^{23}$ Therefore it is difficult to determine the quality of the junctions (i.e., whether the junctions are dominated by defects are not) solely based on $J(V)$ characterization. ${ }^{6,68}$ Recently, we showed that the quality of the junctions can be qualitatively determined by examination the curvature of the $\mathrm{d} J / \mathrm{d} V$ curves. $^{23}$ Here we show that impedance spectroscopy makes it possible to examine the role of defects in molecular junctions and which (circuit) elements are affected. We note, however, that this method only works for large area junctions (junctions with $\gg 1000$ molecules) since small area junctions (or single molecule junctions) have too small capacitances $(\ll 1 \mathrm{pF})$ to be measurable reliably. ${ }^{69}$

Defects induced by the electrode roughness results in an underestimation of the resistance of the SAM complicating quantitative analyses of data obtained from different platforms

The potentiodynamic impedance measurements and equivalent circuit modelling show that only the resistance of the SAM $\left(R_{\mathrm{SAM}}\right)$ is affected due to leakage currents across the SAMs. The contact resistance $\left(R_{\mathrm{C}}\right)$ and capacitance of the junction $\left(C_{\mathrm{SAM}}\right)$ are not sensitive to the surface roughness of the bottomelectrode while the value of $R_{\mathrm{SAM}}$ changed by two orders of magnitude. In other words, defective junctions behave as if they were leaky capacitors and this observation can explain, at least in part, the large spread of current densities measured for the same type of molecules across different test beds. ${ }^{\mathbf{1 4}}$ More specifically, often metal electrodes are used to support SAMs that were obtained by direct deposition techniques, and/or the morphology of the electrodes is not reported. Our results, however, indicate that for quantitative analyses and comparisons of charge transport data across different types of junction platforms, it is important to report the morphology of the electrode materials.

\section{The dielectric constant is insensitive to defects}

The SAM based tunneling junction can be modelled as a parallel plate capacitor with a resistor connected in parallel across which the leakage current through the capacitor flows. For clarity, in this view of the junctions the leakage current across the capacitor is the same as the tunneling current across the SAMs. For a high quality capacitor, the capacitance should be high with a minimal leakage current flowing across it. Here we show that the leakage current through the SAM-based capacitors is sensitive to defects but the dielectric constant is not. This observation explains why the dielectric constant of aliphatic SAMs in other junction platforms based on Hg-drop and PEDOT:PSS electrodes are essentially the same as to the value we report here despite the large difference of the $R_{\mathrm{SAM}}$ and electrode materials between these platforms. ${ }^{45,65}$

\section{Grain boundaries are the main cause of leakage currents in EGaIn junctions}

The TS surfaces have very low amount of step edges or pinholes. ${ }^{60}$ The primary source of defects in our junctions with alkanethiolate SAMs originate from the grain boundaries. Therefore, ultra-flat surfaces (rms surface roughness $<1 \mathrm{~nm}$ ) with large grains help to reduce the density of defects inside the junctions. Vilan et al. achieved grain boundary-free Al films by ultra-fast thermal deposition method. ${ }^{70}$ These surfaces can support alkylphosphonate monolayers with almost no gauche defects. ${ }^{71}$ However, in case of the pre-existing defects due to the fabrication procedures (as is the case for commonly used metal electrodes), the choice of the SAM can also help to reduce the effect of the defects induced by the topography of the electrodes due to self-repair. ${ }^{25}$

\section{Conflicts of interest}

There are no conflicts to declare.

\section{Acknowledgements}

We acknowledge the Ministry of Education (MOE) for supporting this research under award No. MOE2015-T2-2-134. Prime Minister's Office, Singapore under its Medium sized centre program is also acknowledged for supporting this research.

\section{References}

1 A. C. Aragones, D. Aravena, F. J. Valverde-Munoz, J. A. Real, F. Sanz, I. Diez-Perez and E. Ruiz, J. Am. Chem. Soc., 2017, 139, 5768-5778.

2 R. Frisenda, R. Gaudenzi, C. Franco, M. Mas-Torrent, C. Rovira, J. Veciana, I. Alcon, S. T. Bromley, E. Burzuri and H. S. J. van der Zant, Nano Lett., 2015, 15, 3109-3114. 
3 R. Hayakawa, M. A. Karimi, J. Wolf, T. Huhn, M. S. Zollner, C. Herrmann and E. Scheer, Nano Lett., 2016, 16, 4960-4967. 4 D. Taherinia, C. E. Smith, S. Ghosh, S. O. Odoh, L. Balhorn, L. Gagliardi, C. J. Cramer and C. D. Frisbie, ACS Nano, 2016, 10, 4372-4383.

5 J. O. Island, R. Gaudenzi, J. d. Bruijckere, E. Burzurí, C. Franco, M. Mas-Torrent, C. Rovira, J. Veciana, T. M. Klapwijk, R. Aguado and H. S. J. v. d. Zant, Phys. Rev. Lett., 2017, 118, 117001.

6 M. Baghbanzadeh, C. M. Bowers, D. Rappoport, T. Zaba, M. Gonidec, M. H. Al-Sayah, P. Cyganik, A. Aspuru-Guzik and G. M. Whitesides, Angew. Chem., Int. Ed., 2015, 54, 14743-14747.

7 M. Carlotti, A. Kovalchuk, T. Wachter, X. K. Qiu, M. Zharnikov and R. C. Chiechi, Nat. Commun., 2016, 7, 7. 8 R. M. Metzger, Chem. Rev., 2015, 115, 5056-5115.

9 A. Vilan, D. Aswal and D. Cahen, Chem. Rev., 2017, 117, 42484286.

10 G. D. Kong, M. Kim, S. J. Cho and H. J. Yoon, Angew. Chem., Int. Ed., 2016, 55, 10307-10311.

11 H. Jeong, D. Kim, D. Xiang and T. Lee, ACS Nano, 2017, 11, 6511-6548.

12 A. J. Bergren, L. Zeer-Wanklyn, M. Semple, N. Pekas, B. Szeto and R. L. McCreery, J. Phys. Condens. Matter, 2016, 28, 094011.

13 J. C. Love, L. A. Estroff, J. K. Kriebel, R. G. Nuzzo and G. M. Whitesides, Chem. Rev., 2005, 105, 1103.

14 H. B. Akkerman and B. de Boer, J. Phys. Condens. Matter, 2008, 20, 20.

15 D. Xiang, X. Wang, C. Jia, T. Lee and X. Guo, Chem. Rev., 2016, 116, 4318-4440.

16 R. L. McCreery and A. J. Bergren, Adv. Mater., 2009, 21, 43034322.

17 A. P. Bonifas and R. L. McCreery, Nat. Nanotechnol., 2010, 5, 612-617.

18 H. Haick, O. Niitsoo, J. Ghabboun and D. Cahen, J. Phys. Chem. C, 2007, 111, 2318-2329.

19 D. Q. Gao, F. Scholz, H. G. Nothofer, W. E. Ford, U. Scherf, J. M. Wessels, A. Yasuda and F. von Wrochem, J. Am. Chem. Soc., 2011, 133, 5921-5930.

20 H. B. Akkerman, P. W. M. Blom, D. M. de Leeuw and B. de Boer, Nature, 2006, 441, 69-72.

21 L. Yuan, L. Jiang, D. Thompson and C. A. Nijhuis, J. Am. Chem. Soc., 2014, 136, 6554-6557.

22 L. Yuan, L. Jiang, B. Zhang and C. A. Nijhuis, Angew. Chem., Int. Ed., 2014, 53, 3377-3381.

23 L. Jiang, C. S. S. Sangeeth, A. Wan, A. Vilan and C. A. Nijhuis, J. Phys. Chem. C, 2015, 119, 960-969.

24 E. A. Weiss, R. C. Chiechi, G. K. Kaufman, J. K. Kriebel, Z. F. Li, M. Duati, M. A. Rampi and G. M. Whitesides, J. Am. Chem. Soc., 2007, 129, 4336-4349.

25 L. Jiang, C. S. Sangeeth, L. Yuan, D. Thompson and C. A. Nijhuis, Nano Lett., 2015, 15, 6643-6649.

26 L. Jiang, L. Yuan, L. Cao and C. A. Nijhuis, J. Am. Chem. Soc., 2014, 136, 1982-1991.
27 W. J. Hong, D. Z. Manrique, P. Moreno-Garcia, M. Gulcur, A. Mishchenko, C. J. Lambert, M. R. Bryce and T. Wandlowski, J. Am. Chem. Soc., 2011, 134, 2292-2304.

28 A. Mishchenko, D. Vonlanthen, V. Meded, M. Burkle, C. Li, I. V. Pobelov, A. Bagrets, J. K. Viljas, F. Pauly, F. Evers, M. Mayor and T. Wandlowski, Nano Lett., 2010, 10, 156-163. 29 C. Li, I. Pobelov, T. Wandlowski, A. Bagrets, A. Arnold and F. Evers, J. Am. Chem. Soc., 2008, 130, 318-326.

30 B. Q. Xu and N. J. J. Tao, Science, 2003, 301, 1221-1223.

31 S. V. Aradhya and L. Venkataraman, Nat. Nanotechnol., 2013, 8, 399-410.

32 E. S. Juan Carlos Cuevas, Molecular Electronics: An Introduction to Theory and Experiment, World Scientific, Singapore, 2010.

33 C. S. S. Sangeeth, A. Wan and C. A. Nijhuis, J. Am. Chem. Soc., 2014, 136, 11134-11144.

34 K. C. Liao, L. Y. Hsu, C. M. Bowers, H. Rabitz and G. M. Whitesides, J. Am. Chem. Soc., 2015, 137, 5948-5954.

35 J. Sporrer, J. H. Chen, Z. J. Wang and M. M. Thuo, J. Phys. Chem. Lett., 2015, 6, 4952-4958.

36 Z. T. Xie, I. Baldea, A. T. Demissie, C. E. Smith, Y. F. Wu, G. Haugstad and C. D. Frisbie, J. Am. Chem. Soc., 2017, 139, 5696-5699.

37 C. E. Smith, S. O. Odoh, S. Ghosh, L. Gagliardi, C. J. Cramer and C. D. Frisbie, J. Am. Chem. Soc., 2015, 137, 15732-15741.

38 M. Baghbanzadeh, C. M. Bowers, D. Rappoport, T. Zaba, L. Yuan, K. Kang, K. C. Liao, M. Gonidec, P. Rothemund, P. Cyganik, A. Aspuru-Guzik and G. M. Whitesides, J. Am. Chem. Soc., 2017, 139, 7624-7631.

39 K. S. Wimbush, R. M. Fratila, D. D. Wang, D. C. Qi, C. Liang, L. Yuan, N. Yakovlev, K. P. Loh, D. N. Reinhoudt, A. H. Velders and C. A. Nijhuis, Nanoscale, 2014, 6, 1124611258.

40 M. M. Thuo, W. F. Reus, F. C. Simeone, C. Kim, M. D. Schulz, H. J. Yoon and G. M. Whitesides, J. Am. Chem. Soc., 2012, 134, 10876-10884.

41 Q. V. Nguyen, P. Martin, D. Frath, M. L. Della Rocca, F. Lafolet, C. Barraud, P. Lafarge, V. Mukundan, D. James, R. L. McCreery and J. C. Lacroix, J. Am. Chem. Soc., 2017, 139, 11913-11922.

42 C. S. S. Sangeeth, A. Wan and C. A. Nijhuis, Nanoscale, 2015, 7, 12061-12067.

43 C. S. S. Sangeeth, A. T. Demissie, L. Yuan, T. Wang, C. D. Frisbie and C. A. Nijhuis, J. Am. Chem. Soc., 2016, 138, 7305-7314.

44 C. A. Nijhuis, W. F. Reus and G. M. Whitesides, J. Am. Chem. Soc., 2009, 131, 17814-17827.

45 H. B. Akkerman, R. C. G. Naber, B. Jongbloed, P. A. van Hal, P. W. M. Blom, D. M. de Leeuw and B. de Boer, Proc. Natl. Acad. Sci. U. S. A., 2007, 104, 11161-11166.

46 A. Wan, C. S. Suchand Sangeeth, L. Wang, L. Yuan, L. Jiang and C. A. Nijhuis, Nanoscale, 2015, 7, 19547-19556.

47 D. Wang, D. Fracasso, A. Nurbawono, H. V. Annadata, C. S. Sangeeth, L. Yuan and C. A. Nijhuis, Adv. Mater., 2015, 27, 6689-6695.

48 W. F. Reus, M. M. Thuo, N. D. Shapiro, C. A. Nijhuis and G. M. Whitesides, ACS Nano, 2012, 6, 4806-4822. 
49 J. Chen, Z. Wang, S. Oyola-Reynoso and M. M. Thuo, Langmuir, 2017, 33, 13451-13467.

50 F. C. Simeone, H. J. Yoon, M. M. Thuo, J. R. Barber, B. Smith and G. M. Whitesides, J. Am. Chem. Soc., 2013, 135, 1813118144.

51 K. P. McKenna and J. Blumberger, Phys. Rev. B: Condens. Matter Mater. Phys., 2012, 86, 245110.

52 G. Clark, J. R. Schaibley, J. Ross, T. Taniguchi, K. Watanabe, J. R. Hendrickson, S. Mou, W. Yao and X. D. Xu, Nano Lett., 2016, 16, 3944-3948.

53 U. Chandni, K. Watanabe, T. Taniguchi and J. P. Eisenstein, Nano Lett., 2016, 16, 7982-7987.

54 P. Bampoulis, R. van Bremen, Q. R. Yao, B. Poelsema, H. J. W. Zandvliet and K. Sotthewes, ACS Appl. Mater. Interfaces, 2017, 9, 19278-19286.

55 C. A. Nijhuis, W. F. Reus and G. M. Whitesides, J. Am. Chem. Soc., 2010, 132, 18386-18401.

56 H. Haick, J. Ghabboun and D. Cahen, Appl. Phys. Lett., 2005, 86, 042113.

57 C. N. Lau, D. R. Stewart, R. S. Williams and M. Bockrath, Nano Lett., 2004, 4, 569-572.

58 T. W. Kim, G. N. Wang, H. Lee and T. Lee, Nanotechnology, 2007, 18, 315204-315211.

59 J. M. Beebe and J. G. Kushmerick, Appl. Phys. Lett., 2007, 90, 083117.

60 L. Jiang, T. Wang and C. A. Nijhuis, Thin Solid Films, 2015, 593, 26-39.
61 A. Wan, L. Jiang, C. S. S. Sangeeth and C. A. Nijhuis, Adv. Funct. Mater., 2014, 24, 4442-4456.

62 S. Datta, Electronic transport in mesoscopic systems, Cambridge university press, Cambridge, UK, 1995.

63 J. R. Macdonald and W. B. Johnson, Impedance Spectroscopy Theory, Experiment, and Applications, John Wiley \& Sons, Inc., Hoboken, NJ, 2005.

64 D. A. Rabson, B. J. Jonsson-Akerman, A. H. Romero, R. Escudero, C. Leighton, S. Kim and I. K. Schuller, J. Appl. Phys., 2001, 89, 2786-2790.

65 M. A. Rampi, O. J. A. Schueller and G. M. Whitesides, Appl. Phys. Lett., 1998, 72, 1781-1783.

66 R. E. Holmlin, R. Haag, M. L. Chabinyc, R. F. Ismagilov, A. E. Cohen, A. Terfort, M. A. Rampi and G. M. Whitesides, J. Am. Chem. Soc., 2001, 123, 5075-5085.

67 G. Wang, T. W. Kim and T. Lee, J. Mater. Chem., 2011, 21, 18117-18136.

68 E. B. S. Lundqvist, Tunneling Phenomena in Solids, Plenum, New York, 1969.

69 A. Vilan, D. Aswal and D. Cahen, Chem. Rev., 2017, 117, 4248-4286.

70 I. Levine, A. Yoffe, A. Salomon, W. Li, Y. Feldman and A. Vilan, J. Appl. Phys., 2012, 111, 124320.

71 I. Levine, S. M. Weber, Y. Feldman, T. Bendikov, H. Cohen, D. Cahen and A. Vilan, Langmuir, 2012, 28, 404-415. 